\title{
USOS POTENCIALES DEL EFLUENTE DEL HUMEDAL- CONSTRUIDO EN LA PONTIFICIA UNIVERSIDAD JAVERIANA BOGOTÁ
}

\section{POTENTIAL USES OF THE PONTIFICIA UNIVERSIDAD JAVERIANA CONSTRUCTED WETLAND EFFLUENT}

\begin{abstract}
Carolina Ardila-Quintero ${ }^{1}$, Ricardo León-Ramírez ${ }^{2}$, Sandra Galarza-Molina ${ }^{3}$, Andrés Torres ${ }^{4}$
${ }^{1}$ Estudiante Ingeniería Civil, Facultad de Ingeniería. Pontificia Universidad Javeriana, e-mail: ardila.carolina@javeriana.edu. co; ${ }^{2}$ Estudiante Ingeniería Civil, Facultad de Ingeniería. Pontificia Universidad Javeriana, e-mail: r.leon@javeriana.edu.co; ${ }^{3}$ Ingeniera Civil, M.Sc. Hidrosistemas y Estudiante Ph.D. Ingeniería, e-mail: sgalarza@javeriana.edu.co; ${ }^{4}$ Ingeniero Civil, Especialista Sistemas Gerenciales de Ingeniería, M.Sc. Ingeniería Civil y Ph.D. Ingeniería Civil con énfasis en Hidrología Urbana. Grupo de investigación Ciencia e Ingeniería del Agua y el Ambiente, Facultad de Ingeniería. Pontificia Universidad Javeriana, carrera 7 No. 40-62, edificio J.G. Maldonado, S.J., Bogotá, D.C., Colombia, e-mail: andres.torres@javeriana.edu.co
\end{abstract}

Rev. U.D.C.A Act. \& Div. Cient. 19(1): 237-242, Enero-Junio, 2016

\section{INTRODUCCIÓN}

El uso de humedales construidos (HC) como sistemas de aprovechamiento de aguas lluvias (AALL), se ha incrementado en los últimos años, en comparación con otros sistemas convencionales, debido a su fácil operación y mantenimiento y, además, el bajo costo de estos sistemas, promueve su uso en comunidades pequeñas y en países en desarrollo (Kivaisi, 2001; Choi et al. 2012; Zhang et al. 2012). Resultados muestran que la mayor parte del agua lluvia es recolectada en los techos de las casas, implementando sistemas que almacenan agua durante eventos de lluvia (Fletcher et al. 2008; Abdulla \& Shareef, 2009); sin embargo, es primordial la realización de estudios de calidad de aguas para permitir su uso, porque pueden contener contaminantes microbiológicos y químicos (Huston et al. 2009; Moon et al. 2012; Comino et al. 2013; Herrmann, 2012).

Se observa que existen investigaciones en el contexto internacional y nacional sobre el AALL (Torres et al. 2012; Kazemi \& Hill, 2015; Nnadi et al. 2015); no obstante, son escasas las experiencias reportadas en la literatura sobre HC utilizados como elementos de AALL de escorrentía urbana y, la mayoría de investigaciones, no incluye la calidad requerida para usos de agua no potable. De igual forma, se encuentran pocos casos reportados en la literatura sobre $\mathrm{HC}$ en campus universitarios (Mitsch et al. 2008).

Actualmente, en Colombia, el AALL se concentra en experiencias tradicionales de captación y el agua recolectada de los tejados, se emplea para usos no potables (Torres et al.
2012). Respecto a los HC, en Bogotá, han sido empleados para el control de la contaminación proveniente de las aguas de escorrentía de primer lavado, mas no para el AALL (Lara-Borrero, 2010). Durante el 2012 y 2013, la Pontificia Universidad Javeriana Bogotá (PUJB) decidió construir un sistema para el AALL; la tecnología escogida, humedalconstruido/tanque-regulador (HCTR), recoge la escorrentía de un edificio de parqueaderos de la universidad $\left(3.776 \mathrm{~m}^{2}\right)$, de la cancha de fútbol y de las áreas alrededor del sistema $\left(14.816 \mathrm{~m}^{2}\right)$ (Galarza et al. 2015). Este trabajo buscó determinar los posibles usos del agua lluvia que entra y sale del sistema HCTR, por medio de la caracterización del líquido, durante tres eventos de lluvia.

\section{MATERIALES Y MÉTODOS}

Para evaluar la calidad del agua de escorrentía que entra y sale del HCTR de la PUJB, se revisaron estándares de calidad, a nivel nacional e internacional. Se consultaron documentos emitidos por parte del Ministerio de Salud y de Agricultura de Colombia (Colombia, 1984), la Agencia de Protección Ambiental de los Estados Unidos (US-EPA, 2004), el Ministerio de Tierras, Infraestructura y Transporte de Japón (MLIT) (Japón, 2005) y la Organización de las Naciones Unidas para la Alimentación y la Agricultura (FAO) (Pescod, 1992). En estos documentos, se identificaron los límites de los usos proyectados en el campus de la PUJB: riego no agrícola, riego agrícola superficial, lavado de pisos y fachadas, sanitarios, duchas y paisajístico (Tabla 1). 
Tabla 1. Límites recomendados de parámetros de calidad del agua, para determinados usos.

\begin{tabular}{|c|c|c|c|c|}
\hline Parámetros Usos & Sanitarios y Duchas & $\begin{array}{l}\text { Riego agrícola } \\
\text { superficial y no } \\
\text { Agrícola }\end{array}$ & $\begin{array}{l}\text { Lavado } \\
\text { de pisos y } \\
\text { fachadas }\end{array}$ & Uso paisajístico \\
\hline $\mathrm{pH}$ & $\begin{array}{l}6,0-9,0^{1} \\
5,8-8,6\left(\text { Sanitarios }^{2}\right) \\
{ }^{5}, 0-9,03\end{array}$ & $\begin{array}{l}6,0-9,0^{1} \\
<7^{4}\end{array}$ & $5,8-8,6^{2}$ & $\begin{array}{l}6,0-9,0^{1} \\
5,0-9,0^{3}\end{array}$ \\
\hline $\mathrm{DBO}_{5}$ & $<=10 \mathrm{mg} / \mathrm{L}^{1}$ & $\begin{array}{l}<=10 \mathrm{mg} / \mathrm{L} \text { (Riego no } \\
\text { Agrícola }^{1} \text { ) } \\
<=30 \mathrm{mg}^{\prime} \mathrm{L} 1\end{array}$ & & $<=10 \mathrm{mg} / \mathrm{L}^{1}$ \\
\hline SST & $<=5 \mathrm{mg} / \mathrm{L}^{1}$ & $\begin{array}{l}<=5 \mathrm{mg} / \mathrm{L} \text { (Riego no } \\
\text { Agrícola }^{1} \text { ) } \\
<=30 \mathrm{mg} / \mathrm{L}^{1}\end{array}$ & & $<=30 \mathrm{mg} / \mathrm{L}^{1}$ \\
\hline STD & & $<500 \mathrm{mg} / \mathrm{L}^{4}$ & & \\
\hline $\mathbf{T}$ & $\begin{array}{l}<=5 \text { NTU }\left({ }^{\text {Sanitarios }}{ }^{1}\right) \\
<=2 \text { NTU }^{1 \mathrm{y} 2}\end{array}$ & $\begin{array}{l}<=5 \text { NTU (Riego no } \\
\left.\text { Agrícola }^{1}\right)\end{array}$ & $<=2 \mathrm{NTU}^{2}$ & \\
\hline E.coli & $\begin{array}{l}\text { ND } \\
<500 \mathrm{cfu} / 100 \mathrm{~mL}\left(\text { Duchas }^{5}\right)\end{array}$ & $\begin{array}{l}\text { ND (Riego no } \\
\left.\text { Agrícola }^{1}\right) \\
<500 \mathrm{cfu} / 100 \mathrm{~mL} \\
\text { (Riego no Agrícola }^{4} \text { ) }\end{array}$ & & \\
\hline ColiTot & $\begin{array}{l}\mathrm{ND}\left(\text { Sanitarios }^{2}\right) \\
<1000 \\
\text { microorganismos } / 100 \mathrm{~mL}^{3}\end{array}$ & & $\mathrm{ND}^{2}$ & $\begin{array}{l}<5000 \\
\text { microorganismos } / 100 \mathrm{~mL}^{3}\end{array}$ \\
\hline $\begin{array}{l}\text { Población } \\
\text { Bacteriológica }\end{array}$ & & $\begin{array}{l}<10000 \text { individuos/L } \\
\text { (Riego no Agrícola }^{4} \text { ) }\end{array}$ & & \\
\hline $\mathrm{Cl}_{2}{ }^{*}$ & $>=1 \mathrm{mg} / \mathrm{L}^{1}$ & $>=1 \mathrm{mg} / \mathrm{L}^{1}$ & & $>=1 \mathrm{mg} / \mathrm{L}^{1}$ \\
\hline Manganeso (Mn) & & $\begin{array}{l}<0,1 \mathrm{mg} / \mathrm{L} \text { (Riego no } \\
\text { Agrícola }^{4} \text { ) }\end{array}$ & & \\
\hline Hierro $(\mathrm{Fe})$ & & $\begin{array}{l}<0,1 \mathrm{mg} / \mathrm{L} \text { (Riego no } \\
\text { Agrícola }^{4} \text { ) }\end{array}$ & & \\
\hline
\end{tabular}

${ }^{1}$ Agencia de Protección Ambiental de los Estados Unidos (EPA).

${ }^{2}$ Manual de la calidad del agua para la reutilización de las aguas residuales municipales tratadas (MLIT).

${ }^{3}$ Decreto 1594 de 1984 "usos del agua y residuos líquidos" (Colombia).

${ }^{4}$ Organización de las Naciones Unidas para la Alimentación y la Agricultura (FAO).

${ }^{5}$ Estándares de calidad del agua para usos en duchas de la Unión Europea (EU).

* Se debe cumplir este valor de Cloro total residual, después de un contacto mínimo de 30 minutos.

El proyecto de AALL de la PUJB es el resultado de un proceso de investigación desarrollado desde el 2007, por el grupo de investigación Ciencia e Ingeniería del Agua y el Ambiente, con el apoyo de Oficina de Recursos Físicos de la Universidad. El HCTR, se encuentra en el costado norte de la cancha de fútbol del campus de la PUJB, diseñado para mejorar la calidad del agua de escorrentía del edificio de parqueaderos. El sistema recibe caudales entre 0.04-50.6 L/s, puede retener hasta el $75 \%$ del volumen total de entrada y el tiempo de retención en promedio es superior a 20 minutos. El HC es un sistema que posee flujo horizontal sub-superficial; la cama de material granular -con una altura en promedio de 60 $\mathrm{cm}-$, se dividió en tres zonas con diferentes tamaños de grava, para reducir, al mínimo, su obstrucción. El tamaño de la grava varía de forma descendente, empezando en la primera tercera parte, con 1", luego con 3/4" y termina con $1 / 2$ ". El HCTR tiene dos sedimentadores: uno antes del $\mathrm{HC}$ y el otro que reciben la escorrentía de la cancha de fútbol (Galarza et al. 2015). 
Se realizaron tres campañas de muestreo durante tres eventos de lluvia del 2013: 13 de agosto, 17 de septiembre y primero de octubre. Las muestras fueron tomadas en cuatro puntos estratégicos, para evaluar las entradas al HCTR y la salida del sistema: punto 1 , entrada al $\mathrm{HC}$, proveniente del edificio de parqueaderos; punto 2, salida del HC; punto 3, entrada al sedimentador de la cancha de fútbol y punto 4 , salida del sistema HCTR. En los tres primeros puntos, se tomaron seis muestras en cada uno, para ver los cambios de las concentraciones durante cada evento lluvioso. Para el punto 4 , se tomó una muestra, debido a que el agua tratada por el HCTR se utiliza varias horas o, incluso, días después de cada evento.

Durante cada evento, la recolección de muestras se realizó con un intervalo de tres minutos; posteriormente, se realizaron los respectivos ensayos de calidad, $\mathrm{pH}$, turbiedad, sólidos totales -ST-, sólidos suspendidos totales -SST-, sólidos disueltos totales -SDT-, cloruros y demanda bioquímica de oxígeno - $\mathrm{DBO}_{5}$ - siguiendo los protocolos establecidos por el Standard Methods capítulo 1000 edición 22 (Rice et al. 2012); estos ensayos fueron analizados en el laboratorio del Departamento de Ingeniería Civil de la PUJB. Adicionalmente, las muestras del punto 1 y del punto 4 , se sometieron al análisis del número más probable (NMP) de Escherichia coli y coliformes totales y conteo total de población bacteriológica; estos ensayos, se realizaron en la empresa Asebiol LTDA. Cabe resaltar que para el uso de riego no agrícola, las normas de calidad de agua exigen límites de presencia de metales pesados; en este estudio, no se realizaron análisis para dichos metales, por falta de presupuesto.

Para realizar una comparación entre los puntos de muestreo respecto a los indicadores de calidad de agua de los tres eventos, se empleó la prueba estadística t-test.

\section{RESULTADOS Y DISCUSIÓN}

En la tabla 2, se presentan los resultados de calidad en cada punto para los tres eventos lluviosos. En el caso del pH, el punto 1 presentó el mayor valor de $\mathrm{pH}$ en los tres eventos y, el punto 2, el de menores valores. En el segundo evento se observa, a diferencia del primer y tercer evento, que el punto 4 presentó valores más altos de $\mathrm{pH}$ que el punto 3 . La EPA exige pH entre 6 y 9 (Tabla 1) para uso en sanitarios, en duchas, en riego agrícola superficial y no agrícola y paisajístico, todos los puntos cumplieron este parámetro; sin embargo, según la $\mathrm{FAO}$, que exige $\mathrm{pH}$ menor a 7 , en los tres eventos ningún punto cumplió para riego agrícola superficial y riego no agrícola. Finalmente, para el uso lavado, de acuerdo al MLIT (Tabla 1), la mayoría de puntos cumplieron, excepto el punto 1 del primer evento.

Respecto al parámetro turbiedad en los tres eventos, el punto 1 de muestreo presentó los mayores valores y el punto 4 , los menores. Solo el punto 4, en el segundo y tercer evento, respecto a este parámetro, cumple con uso en sanitarios y riego no agrícola, según lo estipulado por la EPA (Tabla 1). El resto de los puntos no cumplen con este parámetro de calidad, para alguno de los otros usos. De acuerdo a los resultados para SST, el punto 1 del primer evento presentó los valores más altos de SST en los tres eventos lluviosos. La concentración de SST en los puntos 2 y 4 presentaron los valores más bajos. Para usos en sanitarios, duchas y riego no agrícola ninguno de los puntos, en los tres eventos lluviosos, cumplió con las normas. Los puntos de muestreo 2 y 3 en los tres eventos, según la EPA, cumplieron para riego agrícola superficial y paisajístico (Tabla 1).

Todos los puntos de muestreo en los tres eventos lluviosos cumplieron con la concentración de STD para riego no agrícola y riego agrícola superficial, según la FAO (Tabla 1). En el primer y tercer evento, los puntos 1 y 2 no presentaron mayor diferencia numérica para STD respecto a los demás indicadores de calidad de agua y los puntos 3 y 4 muestran los máximos valores; sin embargo, en el segundo evento, los puntos 1 y 4 presentaron los mayores valores y, los puntos 2 y 3 , los menores.

Respecto a los resultados de $\mathrm{DBO}_{5}$, en el primer evento, el punto 1 tiene los valores máximos durante los tres eventos. En el segundo punto de muestreo, las concentraciones de $\mathrm{DBO}_{5}$ se mantienen dentro del mismo rango e inferiores a $6,5 \mathrm{mg} / \mathrm{L}$, en los tres eventos. De acuerdo a la EPA, todos los puntos, excepto el punto 1 y 3 del primer evento, cumplen para riego agrícola superficial. El punto 4 del primer evento, los puntos 2 y 4 del segundo evento y todos los puntos del tercer evento, cumplen para uso paisajístico, riego no agrícola, sanitario y ducha, de acuerdo a la EPA, con $\mathrm{DBO}_{5}$ (Tabla 1). Finalmente, para la mayoría de los usos en el segundo y tercer evento, el agua no cumplió con E. coli y coliformes totales.

De acuerdo a los resultados de la prueba t-test, se observó que los valores de turbiedad en el punto 1 del segundo y tercer evento, no presenta diferencias significativas ( $p$-value $>0,05)$, lo mismo sucede con los STD del primer y tercer evento. El segundo punto de muestro, en los tres eventos, es significativamente diferente respecto a turbiedad, SST y cloruros; no obstante, en STD y DBO5 no se presentan diferencias estadísticas ( $p$-value $>0,05$ ). En el punto 3 , todas las concentraciones de los indicadores son significativamente diferentes en los tres eventos; sin embargo, se observa que los resultados de $\mathrm{DBO}_{5}$ del segundo y tercer evento, no presentan diferencias. Para el caso del punto 4, las concentraciones de los parámetros de calidad de agua del primer evento lluvioso son significativamente diferentes del segundo y tercer evento, excepto en SDT, del segundo evento. No se pudo hacer la comparación en cuanto a $\mathrm{DBO}_{5}$ en el punto 4 
Tabla 2. Valores mínimos y máximos de calidad para cada punto y evento.

\begin{tabular}{|c|c|c|c|c|c|c|c|c|}
\hline $\begin{array}{l}\text { Punto- } \\
\text { Evento }\end{array}$ & $\mathrm{pH}$ & $\begin{array}{l}\text { Turbiedad } \\
\text { (NTU) }\end{array}$ & $\begin{array}{c}\text { SST } \\
(\mathrm{mg} / \mathrm{L})\end{array}$ & $\begin{array}{c}\text { SDT } \\
(\mathrm{mg} / \mathrm{L})\end{array}$ & $\begin{array}{c}\text { Cloruros } \\
(\mathrm{mg} / \mathrm{L})\end{array}$ & $\begin{array}{l}\mathrm{DBO}_{5} \\
(\mathrm{mg} / \mathrm{L})\end{array}$ & $\begin{array}{l}\text { Col T } \\
\text { (NMP) }\end{array}$ & E. coli \\
\hline $1-1$ & $8,1-8,9$ & $119-163$ & 896-392 & $20-120$ & $0,3-1,5$ & $29-88$ & 1400 & $\begin{array}{c}16 \\
\text { (UFC/100mL) }\end{array}$ \\
\hline $2-1$ & $6,4-6,8$ & $21-25$ & $0-15$ & $10-100$ & $0,3-1,8$ & $0-4,5$ & & \\
\hline 3-1 & $7,5-7,8$ & $23-150$ & $134-394$ & $160-190$ & $4,0-6,5$ & $8-31$ & & \\
\hline $4-1$ & $7,4-7,5$ & $7,5-7,6$ & $7-10$ & $140-150$ & $1,8-2,5$ & $9-10$ & $<1$ & $\begin{array}{c}<1 \\
(\mathrm{UFC} / 100 \mathrm{~mL})\end{array}$ \\
\hline $1-2$ & $7,2-8,0$ & $8,4-67$ & $7-109$ & $116-160$ & $1,5-3,0$ & $4-7,5$ & 23 & $<3$ (NMP) \\
\hline $2-2$ & $6,8-7,1$ & $11,9-13,5$ & $1-14$ & $52-84$ & $2,0-2,5$ & $0-6,5$ & & \\
\hline $3-2$ & $7,4-7,5$ & $8,15-8,90$ & $5-36$ & $85-105$ & $3,3-4,4$ & $0-12$ & & \\
\hline $4-2$ & $7,8-7,9$ & $3,4-3,5$ & 15,5 & 124 & $1,9-2,0$ & $3-4,5$ & 15 & $<3(\mathrm{NMP})$ \\
\hline $1-3$ & $8,2-8,5$ & $36-50$ & $128-180$ & $63-92$ & $1,5-3,5$ & $14-27$ & 3.6 & $<3(\mathrm{NMP})$ \\
\hline $2-3$ & $6,8-7,3$ & $9,4-15,9$ & $16-24$ & $72-86$ & $2,0-3,5$ & $3-4$ & & \\
\hline $3-3$ & $7,4-7,6$ & $21,4-25,5$ & $28-68$ & $111-136$ & $2,5-3,5$ & $3,5-4,5$ & & \\
\hline $4-3$ & $7,0-7,3$ & $4,2-4,3$ & 12 & $141-144$ & $4,0-4,3$ & 0 & $<3$ & $<3$ (NMP) \\
\hline
\end{tabular}

del tercer evento respecto al primero y al segundo, ya que no se obtuvieron resultados de laboratorio.

Durante este trabajo, se observó que el agua efluente del sistema HCTR cumplió con todas las normas consultadas para uso paisajístico, por ejemplo, el uso en fuentes. Por lo cual, este resultado sugiere que el agua captada, tratada y almacenada por el sistema HCTR puede ser utilizada para este uso, sin ningún tratamiento suplementario. Adicionalmente, se observó que el agua proveniente del campo de fútbol (punto 3) presenta altas concentraciones. La mezcla del agua del punto antes mencionado, con el agua de la salida del HC (punto 2), afecta la calidad del agua efluente del sistema HCTR (punto 4), limitando los usos potenciales. Si se mejora el proceso de sedimentación en el punto 3 , el agua sería adecuada para otros usos.

El punto de muestreo en el sistema HCTR influye significativamente en los resultados de la concentración de algunos indicadores de calidad de agua. Adicionalmente, el agua a la salida del HC es de mejor calidad que el agua de la salida del sistema HCTR. Por lo tanto, el agua a la salida del HC, sin ingresar al tanque de retención, puede ser utilizada para uso paisajístico, sin ningún tratamiento secundario.

Los resultados muestran altos índices de turbiedad y de SST en el agua efluente del sistema HCTR, por lo cual, el agua no cumple para la mayoría de los usos analizados. Respecto a los parámetros microbiológicos, de los tres eventos, en uno se no se halló $E$. coli y coliformes totales y, en los otros, se detectaron alrededor de $15 \mathrm{NMP} / 100 \mathrm{~mL}$ de estos parámetros, por lo que el uso en duchas y en sanitarios no es posible, pero para riego y uso paisajístico, sí cumple. Finalmente, para los tres eventos lluviosos, se observó que las concentraciones de cloruros y SDT se mantienen constantes en la salida del HC, punto 2 y aumentan al salir del sistema HCTR, punto 4. Este resultado sugiere que el proceso que realiza el agua al pasar por el sistema HCTR no determina cambios, en cuanto a concentraciones de cloruros y de SDT.

Para disminuir turbiedad y SST se recomienda implementar sistemas de tratamiento adicionales: aumento del tiempo de retención en el tanque y adición de algún tipo de coagulante (e.g. sulfato de aluminio). Respecto a la disminución de $E$. coli y coliformes totales, se recomienda implementar sistemas de tratamiento alternativos, que permitan disminuir, específicamente, la contaminación microbiana. La $\mathrm{DBO}_{5}$ no es un buen indicador para la contaminación microbiana, porque tiene en cuenta la contaminación orgánica y no es específica para microorganismos patógenos. La aplicación de cloro y el uso de nuevas tecnologías, como la fotocatálisis heterogénea, se podrían utilizar para este fin. Por último, cabe resaltar que para el uso en riego no agrícola, las normas de calidad de agua exigen límites de presencia de metales pesados, tales como hierro, zinc y cobre.

Conflicto de intereses: El manuscrito fue preparado y revisado con la participación de todos los autores, quienes declaramos que no existe conflicto de intereses que ponga en riesgo la validez de los resultados presentados. 


\section{BIBLIOGRAFÍA}

1. ABDULLA, F.; SHAREEF, A. 2009. Roof rainwater harvesting systems for household water supply in Jordan. Desalination (UK). 243(1-3):195-207.

2. CHOI, J.; MANIQUIZ, M.; GERONIMO, F.; LEE, S.; LEE, B.; KIM, L. 2012. Development of a horizontal subsurface $\square$ ow modular constructed wetland for urban runoff treatment. Water Sci. Techn. (UK). 66(9):1950-1957.

3. COLOMBIA. MINISTERIO DE SALUD Y MINISTERIO DE AGRICULTURA. 1984. Decreto 1594 de 1984: usos del agua y residuos líquidos. Diario Oficial 36700 de julio 26 de 1984. Bogotá. Disponible desde Internet en: http://www. alcaldiabogota.gov. co/sisjur/normas/Norma1.jsp?i=18617 (con acceso 11/08/2013).

4. COMINO, E.; RIGGIO, V.; ROSSO, M. 2013. Grey water treated by an hybrid constructed wetland pilot plant under several stress conditions. Ecol. Engineering. (USA). 53:120-125.

5. FLETCHER, D.; DELETIC, A.; MITCHELL, V.; HATT, B. 2008. Reuse of urban runoff in Australia: A review of recent advances and remaining challenges. J. Environ. Qual. (USA). 37:S-116-S-127.

6. GALARZA-MOLINA, S.; TORRES, A.; LARA-BORRERO, J.; MÉNDEZ-FAJARDO, S.; SOLARTE, L.; GONZALES, L. 2015. Towards a constructed wetland/ reservoir-tank system for rainwater harvesting in an experimental catchment in Colombia. Rev. Ing. Univ. (Colombia). 19(2):169-185.

7. HERRMANN, J. 2012. Chemical and biological benefits in a stormwater wetland in Kalmar, SE Sweden. Limnologica. (Germany). 42(4):299-309.

8. HUSTON, R.; CHAN, Y.; GARDNER, T.; SHAW, G.; CHAMPAN, H. 2009. Characterization of atmospheric deposition as a source of contaminants in urban rainwater tanks. Water research. (Netherlands). 43(6):1630-1640.

9. JAPÓN. MLIT -MINISTRY OF LAND, INFRASTRUCTURE, AND TRANSPORTATION-. 2005. Manual on water quality for reuse of treated municipal wastewater, Tokyo.
10. KAZEMI, F.; HILL, K. 2015. Effect of permeable pavement base course aggregates on stormwater quality for irrigation reuse. Ecol. Engineering. 77:189-195.

11. KIVAISI, A. 2001. The potential for constructed wetlands for wastewater treatment and reuse in developing countries: a review. Ecol. Engineering. 16(4):545560.

12. LARA-BORRERO, J. 2010. Humedales construidos para el control de la contaminación proveniente de la escorrentía urbana. Rev. Acodal. 226(1):19-27

13. MITSCH, W.; TEJADA, J.; NAHLIK, A.; KOHLMANN, B.; BERNAL, B.; HERNANDEZ, C. 2008. Tropical wetlands for climate change research, water quality management and conservation education on a university campus in Costa Rica. Ecol. Engineering. 34(4):276-288.

14. MOON, S.; LEE, J.; LEE, B.; PARK, K.; JO, Y. 2012. Quality of harvested rainwater in artificial recharge site on Jeju volcanic Island, Korea. J. Hydrology. (Germany). 414-415:268-277.

15. NNADI, E.O.; NEWMAN, A.P.; COUPE, S. J.; MBANASO, F.U. 2015. Stormwater harvesting for irrigation purposes: An investigation of chemical quality of water recycled in pervious pavement system. J. Environ. Manag. (Belgium). 147:246-256.

16. PESCOD, M. 1992. Wastewater treatment and use in agriculture: Irrigation with wastewater. Roma, Italy: FAO. Disponible desde Internet en: http://www.fao. org/docrep /t0551e/ t0551e00.HTM (con acceso 12/08/13).

17. RICE, E.W.; BRIDGEWATER, L.; AMERICAN PUBLIC HEALTH ASSOCIATION; AMERICAN WATER WORKS ASSOCIATION; WATER ENVIRONMENT FEDERATION. 2012. Standard methods for the examination of water and wastewater. Ed. APHA-AWWA-WEF (Washington D.C.). 1496p.

18. TORRES, A.; MENDEZ, S.; LARA, J.; ESTUPIÑAN, J.; ZAPATA, H.; TORRES, O. 2012. Hacia equipamientos urbanos sostenibles: aprovechamiento de aguas lluvias en el campus de la Pontificia Universidad Javeriana en Bogotá. Cuadernos de Vivienda y Urbanismo. (Colombia). 5(9):124-141.

19. US-EPA -United States Environmental Protection Agency-. 2004. Guidelines for water reuse. Washington. 
Disponible desde Internet en: Agosto 11 de 2013: http://water.epa.gov/aboutow/owm/upload/WaterReuse-Guidelines-625r04108.pdf (con acceso 11/08/2013).

20. ZHANG, T.; XU, D.; HE, F.; ZHANG, Y.; WU, Z. 2012. Application of constructed wetland for water pollu- tion control in China during 1990-2010. Ecological Engineering. (USA). 47:89-197.

Recibido: Diciembre 17 de 2015

Aceptado: Abril 25 de 2016

\section{Cómo citar:}

Ardila-Quintero, C.; León-Ramírez, R.; Galarza-Molina, S.; Torres, A. 2016. Usos potenciales del efluente del humedal-construido en la Pontificia Universidad Javeriana Bogotá. Rev. U.D.C.A Act. \& Div. Cient. 19(1): 237-242. 